\title{
EL IMPACTO DE LA EXCLUSIÓN EN LA SALUD: UNA FORMA DE VIOLENCIA ESTRUCTURAL.
}

\author{
DANIEL LA PARRA CASADO
}

Depto. Sociología II, Didáctica, Comunicación y Psicología. Universidad de Alicante.

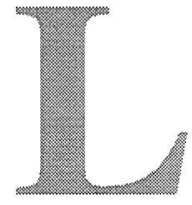

os estudios sobre desigualdades sociales en salud muestran sistemáticamente que los individuos con menores niveles de ingreso, educación, peor situación ocupacional o que viven en las regiones más pobres presentan peores indicadores de salud (Townsend y Davidson, 1982; Navarro y Benach, 1996; Acheson, 1998; Marmot y Wilkinson, 1999; Regidor et al. 1994). Dichos estudios muestran que existe un gradiente en la relación entre estado de salud y posición socioeconómica (la cual suele estar relacionada con factores como la clase social, el género, la etnia, la edad o el lugar de residencia). Pero además de los efectos generales de la desigualdad en la salud, también se pueden describir efectos de la exclusión social en el estado de salud.

A continuación se exponen una serie de datos que pueden informar sobre la influencia directa de la exclusión social en el estado de salud, así como posibles líneas explicativas de los mismos surgidas a partir de una investigación cualitativa a partir de entrevistas en profundidad a mujeres en situación de pobreza. Se avanza que las situaciones de exclusión social tienen un impacto mensurable y directo sobre el estado de salud y el bienestar de la población. Dicho impacto se traduce concretamente en mayor propensión a la enfermedad, en una menor esperanza de vida y en un peor nivel de bienestar, es decir, la exclusión social tiene efectos directos sobre el organismo, lo que acerca los efectos de la violencia estructural (la exclusión) a los efectos de la violencia directa.

Algunos datos empíricos pueden servir para hacer perceptible el impacto de la exclusión social en el estado de salud. Borrell et al. (1997) muestran que el número de años potenciales de vida perdidos por 100.000 habitantes de 1 a 70 años en los barrios más desfavorecidos de Barcelona es un 79\% más alto que en el resto de los barrios de la ciudad y que la diferencia ha crecido desde 1983 a 1994. El SIDA y la 
sobredosis se destacan como las causas de muerte que están contribuyendo en mayor medida al incremento de las desigualdades en la mortalidad prematura. Resultados similares se reproducen en otros lugares y ciudades del mundo (Dorling et al, 2000). En Estados Unidos, por ejemplo, se observa que los afroamericanos residentes en las ciudades sufren tasas muy altas de mortalidad en comparación con la media del país, diferencia que se acentúa entre aquellos que viven en los barrios más pobres (Geronimus et al., 1999). En general se puede afirmar que unos pocos barrios de las ciudades pueden concentrar mayor proporción de muertes por homicidio, accidentes, SIDA, intoxicación, suicidio y los escasos brotes de enfermedades infecciosas, pero también mayores tasas de mortalidad por enfermedad cardiovascular y enfermedades respiratorias.

Esta situación no se encuentra únicamente al comparar barrios. En la mayoría de los estudios sobre desigualdad social, se suelen comparar los resultados entre personas clasificadas en distintas categorías ocupacionales. Habitualmente un grupo de personas no puede ser clasificada en función de la ocupación, de todos modos en ocasiones estos resultados se presentan agrupados en una categoría que actúa como "cajón de sastre" a la que se suele denominar "otros" o "sin clasificar". Estas personas, definidas por tanto, por su exclusión del mercado de trabajo suelen tener peores indicadores de salud que el resto de los grupos comparados. En Annandale (1998), por ejemplo, se puede ver que la mortalidad estandarizada de las ocupaciones no manuales en hombres es de 84, frente a un 103 en clases manuales y un 189 en "otros". Lo mismo ocurre con las mujeres para las que esas cifras son respectivamente de 78, 96 y 106. También en Annandale (1998; 106) con datos longitudinales referidos a la mortalidad masculina en el Reino Unido de personas de 15 a 64 años clasificados por ocupación en 1971 y fallecidos en el periodo 1976 a 1981 se observa que la tasa estandarizada de mortalidad para la clase I (formada por profesionales liberales y propietarios de empresas, según la clasificación del British General Registrar) es igual a 64, mientras que es de 124 para la clase $\mathrm{V}$, de 173 para un grupo clasificado como "descritos de forma inadecuada" y de 212 para los desocupados. Östberg (1997) tomando como referencia al grupo intermedio dentro de las profesiones no manuales encuentra una odds ratio en la mortalidad por enfermedad en niños de 0 a 12 años en el período 1980-1986 en Suecia para el grupo de las profesiones manuales calificadas igual a 1'60. En el grupo "otros" la odds asciende a $2^{\prime} 00\left(\mathrm{p}<0^{\prime} 001\right)$, lo que supone que se duplica el riesgo de las clases sociales intermedias. Leon (1992) et al. obtienen que el grupo social con mayores tasas de mortalidad infantil son aquellos a cuyos padres no se les ha podido atribuir una ocupación. En concreto en Inglaterra y Gales el grupo de niños nacidos tiene un riesgo de mor- 
talidad neonatal igual a 761 por 100.000 nacidos vivos, mientras que los de clase social I tienen un mortalidad neonatal de 432 por 100.000 y los de clase social V de 663 por 100.000 nacidos vivos.

En la mayor parte de la literatura sobre desigualdades en salud la preocupación principal ha sido mostrar la existencia de una asociación, comprobable en términos estadísticos, entre una determinada variable que indica la posición socioeconómica y algún indicador de salud. El desarrollo teórico para explicar los mecanismos por los que dichas variables se convierten en una menor salud ha sido mucho menos frecuente. En este campo se han encontrado varias corrientes, desde quienes han apostado por dar protagonismo a los aspectos materiales, como el nivel de ingresos o el tipo de ocupación que se desempeña (Lynch et al, 2000a y 2000b), a quienes han otorgado un mayor protagonismo a aspectos psicosociales (Wilkinson, 1996).

En cualquier caso estos trabajos se centran en la descripción del gradiente provocado en el estado de salud por la posición socioeconómica, pero no se describen los efectos concretos de la exclusión en la salud. Este el objetivo de esta comunicación.

Para ello se parte de la información obtenida en la realización de entrevistas en profundidad con contenidos biográficos a un total de 55 mujeres dispersas por todo el territorio nacional siguiendo como criterio primordial en la selección de participantes el estar viviendo en una situación de pobreza o precariedad social. Para acceder a la población se contactó con la red de Cáritas España. El método de selección fue de tipo no probabilístico. El propósito perseguido no era tanto conseguir una muestra aleatoria representativa de la población (objetivo inalcanzable cuando se trabaja con una cincuentena de casos), como identificar situaciones de vida específicas que pudieran ser relevantes para el fenómeno estudiado. Se realizaron entrevistas en Andalucía, Extremadura, Madrid, Galicia, Castilla y León, País Vasco, Navarra, Cataluña y Comunidad Valenciana. A los profesionales y trabajadores sociales de Cáritas se les pidió diferentes perfiles de mujeres a entrevistar: diferentes edades (jóvenes, adultas y ancianas) y diferentes situaciones familiares (solteras, casadas, viudas, madres solteras). El resultado final da lugar a una población de estudio compuesta por mujeres en diferentes tipos de situación familiar: madres solteras; mujeres casadas o viudas y un conjunto de mujeres en situaciones familiares altamente inestables y cambiantes (pisos de acogida, rupturas y reconciliaciones). Las participantes en la investigación reflejan situaciones muy distintas de empobrecimiento. Nos encontramos con casos de pobreza tradicional y de nueva pobreza, situaciones de miseria y de movimientos entre una situación estable y precaria. Los motivos de empobrecimiento son muy variados: desde el nacimiento en la pobreza, hasta las 
dificultades de empleo, pasando por la pérdida de bienestar material debido a la enfermedad, las migraciones, la disolución o el conflicto familiar, la drogadicción y la prostitución.

En el análisis de las entrevistas se extrajeron numerosas ideas. Aquí se procuran resumir todos aquellos aspectos relacionados con la descripción del contexto y dimensiones de la exclusión social que pueden estar relacionados con un acceso diferenciado a los recursos de los que depende la salud, ya sean éstos de tipo material, informativos, psíquicos o sociales. La pregunta que ha guiado el trabajo de investigación es cómo se traduce la exclusión social en un factor restrictivo de la salud.

Como todo trabajo de tipo cualitativo, con carácter exploratorio, el análisis de las entrevistas ha servido para extraer una serie de hipótesis que podrían explicar dicha relación. Se han seleccionado aquéllas que describen situaciones que afectan al conjunto de mujeres, dejando de lado, en esta ocasión, las situaciones específicas de grupos concretos (personas con toxicomanías, seropositivas u otros). Estos son algunos de los principales factores que pueden explicar la peor situación de salud:

1. La propia definición de salud que realizan las personas entrevistadas. Por salud se entiende contar con la capacidad de poder trabajar - "yo de salud también estoy bien, que puedo trabajar" $(\mathrm{M} 24)^{1}$ - Este concepto toma prioridad sobre cualquier otra consideración relativa por ejemplo a la salud emocional o a indicadores como el colesterol o la hipertensión. En ocasiones, esta visión funcional de la salud llega a identificar la enfermedad únicamente con haber estado hospitalizada. A la pregunta: «la última vez que estuviste enferma ¿cuándo fue?», contesta una mujer: "es que no he estado nunca ingresada, ni nada» (M17). En general, se observa que el grupo de mujeres entrevistadas se define como sana mientras tiene capacidad de realizar tareas. Las posibles consecuencias para la salud de una definición funcional de la salud son evidentes: descuido de las prácticas preventivas, retraso en el reconocimiento de los síntomas, fuerte presión para acortar el periodo de rehabilitación o negarse a recibir tratamiento si implica reducción de la actividad.

2. La forma de entender definir la propia salud se deriva de la propia situación de precariedad económica. Para alguien que trabaja limpiando casas sin contrato no existe posibilidad de realizar una parada en la actividad al menos que se acuse un problema de salud lo

1 Esta notación se utiliza para referirse a las personas entrevistadas, cuando se extraen citas literales de las entrevistas. 
suficientemente grave ("porque trabajas y si no tienes nómina..., el que no trabaja, no cobra. Yo no puedo permitirme estar enferma. Es triste que viva así, que viva así, pero así fue mi vida"). Algunos relatos muestran en qué medida esto es cierto. Mujeres que han padecido algún problema de salud han perdido su puesto de trabajo y con él, la única fuente de ingresos que entraba en el hogar.

3. La falta de adaptación de las soluciones previstas por el sistema sanitario a las situaciones específica que afectan a la población en situación de exclusión social. Un caso extraído de las entrevistas puede ilustrar esta situación. Se trata de una mujer que opera a su hijo de vegetaciones y que encuentra que éstas se vuelven a reproducir en un breve espacio de tiempo. El médico sugiere una nueva intervención y la madre se niega por considerar que éste se equivoca pues la anterior intervención no ha surtido efecto. En realidad, a lo largo de la entrevista se comprueba, por la descripción que la mujer realiza de su casa, que el hijo duerme en una habitación con muy graves problemas de humedad y que se trata de una casa fría y sombría con un sistema de calefacción muy deficiente que provoca al niño continuas complicaciones respiratorias y repetidos tratamientos con antibióticos. Seguramente la intervención que el niño necesita es vivir en una casa con unas condiciones de habitabilidad mínimas. Situaciones análogas se encuentran, por ejemplo, en tratamientos psiquiátricos a base de ansiolíticos a mujeres cuyo problema es que padecen malos tratos o cuando se pretende dar soluciones médicas a problemas sociales. Una mujer señala que lo que necesita para evitar la depresión y sentirse bien es encontrarse con un buen trabajo ("esas situaciones (se refiere a la escasez económica y falta de trabajo) te crean un estado de ansiedad, de estrés, de... de malestar general. Entonces yo cuando estoy trabajando y me siento con mi dinero, que yo gano, estoy tan a gusto $y$ tan feliz, como me está pasando ahora mismo, que me encuentro a gusto y feliz" (M21)).

4. La importancia de las responsabilidades familiares. Se ha comentado la dificultad de atender a la propia salud como consecuencia de la debilidad de la relación laboral en empleos como el trabajo doméstico o en cualquier otro tipo de actividad sumergida o con contratos precarios. Dicha situación puede afectar al retraso en acudir a la consulta y a la hora de seguir las prescripciones médicas, sobre todo, si éstas aconsejan reposo. Pero no sólo la actividad laboral condiciona el proceso de rehabilitación de la enfermedad. En el caso de las mujeres también son destacables las demandas por parte de la familia. Lo ilustra un caso de una mujer con meningitis. Ella 
relata: "estuve quince días ingresada, yo firmé mi alta porque no podía, no podía permitirme estar más, yo si me muero que me muera en camino, pero mis hijos tengo que... de aquellos mis hijos en (no se entiende) tuvieron a un colegio interno" "con miedo de si me los daban en adopción, alguien, yo tuve que salir" (M34). La presión de las demandas familiares se deja sentir en la mayoría de los relatos. Es especialmente evidente en los casos de hogares monoparentales, pero también se deja sentir en los hogares con apariencia de familia nuclear. En muchos de ellos, la responsabilidad sobre los hijos y otras personas dependientes sigue recayendo sobre las mujeres ("soy yo la que tiene que mirar por mis hijos y por mi casa, no puedo estar pensando en él" "porque soy yo la que lleva la casa").

5. La situación de dependencia económica y vulnerabilidad, tanto en términos materiales como emocionales (en las entrevistas se observan casos de dependencias afectivas y falta de autoestima notables) impiden la posibilidad de desarrollar estilos de vida elegidos y saludables. Ello es patente en las situaciones de malos tratos, que son vividas de modo tan perjudicial y prolongado en el tiempo (a veces la decisión de abandonar se toma pasado lustros, en otros casos no se llega a tomar, otras veces se vuelve con el maltratador) debido a las escasas alternativas vitales disponibles ("por el miedo a que decía a que me iba a quitar los hijos, verme sola, sin un trabajo y decir ¿dónde voy? si no tengo nada", M22).

\section{CONCLUSIÓN.}

Estos breves ejemplos, cabría enumerar muchos más factores, sirven para considerar que las vidas de las personas excluidas transcurren dentro de unos marcos muy determinados por sus propias condiciones socioeconómicas. De manera que la ausencia de oportunidades vitales (empleo de calidad, educación, participación, acceso a los servicios sociales y sanitarios) provoca por vías directas o indirectas una reducción de las posibilidades de disfrutar una vida saludable.

\section{BIBLIOGRAFÍA.}

ACHESON, D. (1998): Independent Inquiry into Health Inequalities Report. London: The Stationary Office.

ANNANDALE, E. (1998): The sociology of Health and Medicine. A critical introduction. Malden: Blackwell and Polity Press. 
BOOTH, S. (1999): Researching Health and Homelessness: Methodological Challenges for Researchers Working with a Vulnerable, Hard to Reach, Transient Population. Australian Journal of Primary Health, 5, 3: 76-81.

BORRELL, C. y ARIAS, A. (1993): Desigualdades de mortalidad en los barrios de Barcelona, 1983-1989. Gaceta sanitaria, 7: 205-220.

BORRELL, C.; PLASÈNCIA, A.; PASARÍN, I. y ORTÚN, V. (1997): Widening social inequalities in mortality: the case of Barcelona, a southern European city. Journal of Epidemiology and Community Health, 51: 659-667.

FARMER, P. (1997): Ethnography, social analysis, and the prevention of sexually transmitted HIV infections among poor women in Haiti», en Inhorn, MC y Brown PJ (eds.) The Anthropology of Infectious Disease. Amsterdam: Gordon and Breach: 413-438.

GERONIMUS, A.T.; BUND, J. y WAIDMANN, T.A. (1999): Poverty, time and place: variation in excess mortality across selected US populations, 19801990. Journal of Epidemiology and Community Health, 1999; 53: 325-334.

GREEN, J. (1999): Commentary: generalisability and validity in qualitative research. British Medical Journal, 319, 1999: 421.

HELMAN, C.G. (1994): Culture, Health and Illness. Oxford: Butterworth Heinemann, third edition.

KLOUDA, A. (1993): Prevention is still more costly than cure, in Reaching Health for All. Dehli: 11-27, ch. 1.

LEON, D.A.; VAGERO, D. y OTTEBLAD OLAUSSON, P. (1992): Social class differences in infant mortality in Sweden. A comparison with England and Wales. British Medical Journal, 305: 687-691.

LYNCH, J.; DUE, P.; MUNTANER, C.y DAVEY SMITH, G. (2000): Social Capital -Is it a good investment strategy for public health? Journal of Epidemiology and Community Health, 54: 404-408.

LYNCH, J.; DAVEY SMITH, G.; KAPLAN G. y HOUSE, J.S. (2000): Income inequality and mortality: importance to health of individual income, psychosocial environment, or material conditions. British Medical Journal, 320: $1200-4$.

MANZA, J. (2000): Race and the underdevelopment of the American welfare state. Theory and Society, 29: 819-832.

MARMOT, M. y WILKINSON, R.G. (1999): Social Determinants of Health. Oxford: Oxford University Press.

NAVARRO, V. y BENACH, J. (1996): Informe de la Comisión Científica de Estudios de las Desigualdades Sociales en Salud en España. Revista de Salud Pública, 70, 5-6, 1996: 505-636.

NAVARRO, V. y SHI, L. (2001): The political context of social inequalities and health. Social Sciences and Medicine, 52: 481-491. 
ÖSTBERG, V. (1997): The social patterning of child mortality: the importance of social class, gender, family structure, immigrant status and population density. Sociology of Health and Illness, 19: 415-435.

REGIDOR, E.; GUTIÉRREZ-FISAC, J.L. y RODRÍGUEZ, C. (1994): Diferencias $y$ desigualdades en salud en España. Madrid: Diaz de Santos.

TOWNSEND, P. y DAVIDSON, N. (1982): Inequalities in health. The Black Report. London, Penguin.

WILKINSON, R.G. (1996): Unhealthy societies. The Afflictions of Inequality. London, Routledge.

WILKINSON, R. y MARMOT, M. (1998): Social Determinants of Health. The solid facts. World Health Organization. 